\section{Cayetano Heredia: una visión contemporánea}

\section{Cayetano Heredia: A contemporary view}

Los discípulos son la biografía del maestro. Domingo Faustino Sarmiento

\section{Sr. Editor:}

Cayetano Heredia es un símbolo de la medicina peruana. Fue el principal continuador de Hipólito Unánue en la organización de los estudios de medicina en Perú.

Nació en 1797, en Piura. Su familia era de condición económica baja. A los diez años llegó a Lima, y a los 15 años fue admitido en el Real Colegio de Medicina y Cirugía de San Fernando. Dos rectores de este colegio lo acogieron como su protegido: el sacerdote Fermín de Goya, y luego don Javier de Luna Pizarro ${ }^{1}$. Su trayectoria estudiantil transcurrió entre el antiguo régimen virreinal y la naciente República del Perú. Heredia sirvió con ardor a las ideas emancipadoras. En 1823 fue nombrado, aún siendo alumno, Disector de Anatomía del Colegio de la Independencia. En 1826, se graduó de médico. Por su conocimiento de anatomía se dedicó a la cirugía, participó como cirujano del ejército en el combate de Huailacucho (1834), bajo las órdenes del general Orbegoso. Fue ascendido al cargo de "Cirujano Jefe" e Inspector General de Hospitales por el mismo Orbegoso. Heredia tenía evidentes vínculos con el ejército, su labor militar fue reconocida en 1836, nombrándolo miembro de la Legión de Honor².

Hipólito Unánue había creado el Real Colegio de Medicina y Cirugía de San Fernando, nombre que se cambió a Colegio de la Independencia. En 1834, el General Santa Cruz, lo nombró Rector de este Colegio $^{1}$. En 1839, por razones políticas, Heredia fue destituido del cargo. La situación del Colegio no mejoró y en 1842 Heredia fue nuevamente llamado a la Dirección. En 1846 fue nombrado Protomédico General de la República ("contralor" de la profesión médica), cargo que junto con el de Decano convirtió a Heredia en la autoridad suprema en educación médica y utilizó ese poder para construir una nueva escuela médica peruana. Sin embargo, para algunos era considerado un dictador ${ }^{1}$.

En 1856, Cayetano Heredia fundó la nueva
Facultad de Medicina, sobre la base del antiguo Colegio de la Independencia, que se reintegró definitivamente a la Universidad de San Marcos tomando el nombre de Facultad de Medicina de San Fernando. Cayetano Heredia fue el primer Decano de la Facultad de Medicina de San Fernando 3 (Figura 1).

Hipólito Unánue y Cayetano Heredia mantuvieron una relación estrecha. Unánue encargó a Heredia el difícil trabajo de realizar una autopsia médico legal al joven prócer de la Independencia, Faustino Sánchez Carrión, que falleció súbitamente, y se sospechaba de un envenenamiento. Heredia concluyó que se trataba de una "ruptura de un aneurisma de la vena hepática" en un contexto de un hígado cirrótico ${ }^{4}$, aunque Ricardo Palma en su epistolario refiere que Heredia tenía dudas sobre su propia conclusión. Heredia fue uno de los pioneros de la medicina legal en Perú.

Con Heredia se produjo una revolución en la escuela de medicina, tanto en su rigor científico como administrativo. Contó con la colaboración de distinguidos profesores extranjeros. Sin em-

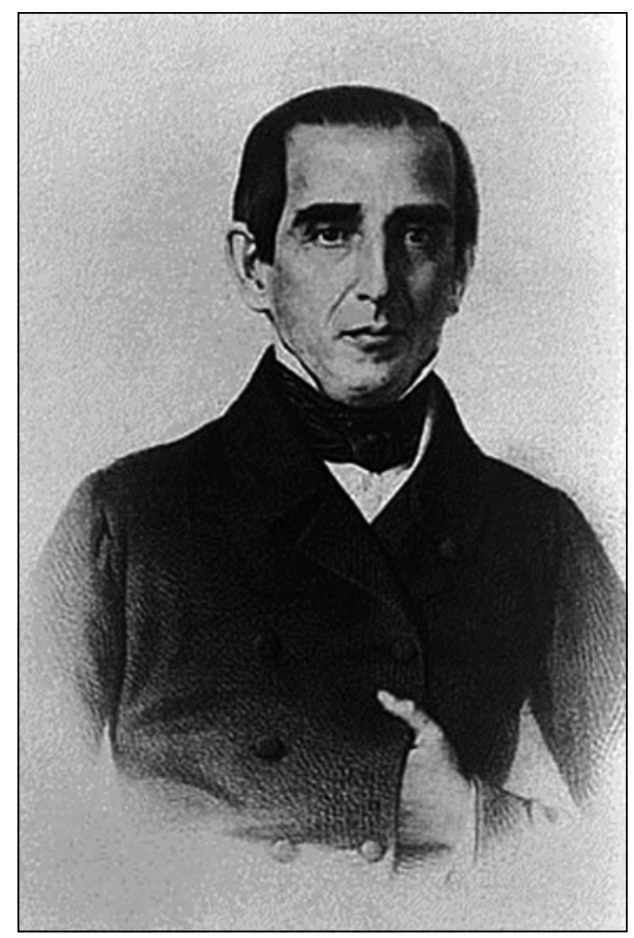

Figura 1. Cayetano Heredia: símbolo de la medicina peruana. Fuente: Lastres J. Historia de la Medicina Peruana, vol. III. La Medicina en la República, 1951. 
bargo, comprendió que debía tener profesores propios, por lo que, con su propio dinero, envió varios médicos jóvenes a la Escuela de París, por cuatro años, luego regresaron como docentes y contribuyeron de manera decidida a las reformas propuestas por Heredia, con los profesores extranjeros Manuel Solari, José Eboli, Antonio Raimondi, Sebastián Lorente, lo que llevó a la Facultad a sus tiempos de mayor esplendor. La Facultad de Medicina de Lima, en 1856, fue modelada a semejanza de la Escuela de París ${ }^{4}$. A diferencia de sus contemporáneos, Heredia modernizó la medicina peruana, incorporando los conocimientos médicos extranjeros, comprendió que la ciencia es necesariamente internacional. El naturalista Raymondi, nombró en su homenaje a una planta: genciana herediana.

El microscopio fue inventado en 1590, inició una revolución científica en Europa y también lo haría en Perú. En 1856 Heredia trajo el primer microscopio a Lima, desde Francia ${ }^{5}$, siendo una de sus obras poco resaltadas. El microscopio fue el instrumento silencioso del cambio científico que buscaba Heredia, contribuyó a que en el Perú se consolidara la investigación científica. Heredia, además, formó la Sociedad Médica de Lima; con sus propio recursos formó en 1845 el gabinete de Física e Historia Natural, luego la Biblioteca del Colegio. Los médicos estaban desprestigiados, casi en la categoría de curanderos. Heredia canceló la vieja estratificación de las profesiones médicas: Físicos, Cirujanos Latinos, Cirujanos Romancistas y Flebótomos. El Colegio de Medicina sólo formaría médicos-cirujanos de la más elevada calidad posible.

Casimiro Ulloa fue el principal alumno de Heredia, enviado por él a Francia, y continuó la obra docente de Heredia fundando la Academia Nacional de Medicina, vigente hasta la actualidad.

Heredia recibió varios ataques. Probablemente uno de ellos se basó en un error suyo. En 1849, dado el alto nivel intelectual de un alumno, Sebastián Barranca, propició su rápida matrícula directamente en el segundo año de medicina. Heredia fue criticado duramente, el alumno Barranca fue expulsado de la Facultad de Medicina. Cayetano Heredia, involuntariamente, por hacerle un bien terminó dañándolo: Barranca nunca llegó a ser médico. Los enemigos de Cayetano Heredia quisieron usar este incidente para expulsarlo. Barranca se dedicó a las Ciencias Naturales ${ }^{6}$ y años después sería el sabio formador de dos símbolos científicos de Perú: Daniel Alcides Carrión y Julio C. Tello.

Heredia fue retirado de su cargo en 1860 y falleció en Lima un año después. En homenaje a él lleva su nombre una de las más famosas universidades peruanas: la Universidad Peruana Cayetano Heredia.

Heredia no realizó publicaciones científicas. Sin embargo, trascendió por su labor en educación médica, y será recordado -como todo educadorpor las obras de sus alumnos.

David Salinas Flores

\section{Referencias}

1. Ulloa J. Elogio de Cayetano Heredia. Acta Herediana 2012; 50: 7-11.

2. Zárate E. Cayetano Heredia: el punto de partida y una dilatada enfermedad. An Fac Med 2013; 74 (3): 249-54.

3. Zárate CE. Inicios de la Escuela de Medicina de Lima. Cayetano Heredia el organizador. 1 ${ }^{a}$ Edición. Lima, Perú. Asamblea Nacional de Rectores. Lima, 2004.

4. Lastres J. Capítulo XXIII. Cayetano Heredia En: Historia de la Medicina Peruana, vol. III. La Medicina en la Re-

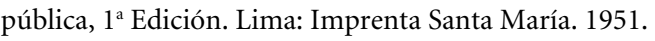

5. García-Cáceres U. Aspectos de la Historia de la Medicina del Perú durante la fundación de la Escuela de Medicina Cayetano Heredia y su tiempo (1797-1861) (Parte I). Acta Med peruana 2011; 28 (2): 112-7.

6. Barranca S. Autobiografia. En Ruiz Alarco F Barranca. Biografía Tomo I Editorial Universitaria. Lima. 1961. págs 57-63.

Correspondencia a:

David Salinas F.

Federico Villarreal 592 Urb Ingeniería San Martín De Porres Lima, Perú

dsalinas2009@yahoo.com 